\title{
EFEITOS DA APLICAÇÃO DE DESSECANTES NA PRODUÇÃO E QUALIDADE DAS SEMENTES DE SOJA EM UBERLÂNDIA-MG
}

\author{
Carlos M. dos Santos ${ }^{1}$, Paulo César Ferruzi², \\ Joaquim Antônio Carvalho ${ }^{1}$ V Vera Lúcia M. dos Santos ${ }^{1}$
}

\footnotetext{
Universidade Federal de Uberlândia - Departamento de Agronomia Uberlândia, MG 38400-902

2Cerragro Agrícola Produtos Agropecuários Ltda. Uberlândia, MG 38400-328
}

\begin{abstract}
RESUMO
Foram conduzidos dois ensaios, o primeiro em campo, numa área comercial de produção de sementes de soja em Uberlândia-MG e o segundo no Laboratório de Sementes da Universidade Federal de Uberlândia, tendo como objetivo avaliar o efeito da aplicação e de doses de dessecantes na produtividade e na qualidade das sementes de soja, cultivar Savana. O delineamento experimental foi de blocos casualizados, com quatro repetições e os tratamentos constituídos dos seguintes produtos e doses: 1) testemunha (colhida na maturação fisiológica), 2) glyphosate-360 (0,36 kg/ha), 3) glyphosate-360 $(0,72$ $\mathrm{kg} / \mathrm{ha}), 4)$ glyphosate-720 (0,36 kg/ha) 5) gyphosate-720 (0,72 kg/ha); 6) glyphosate-540 (0,36 kg/ha) 7) glyphosate-540 $(0,72 \mathrm{~kg} / \mathrm{ha}) 8)$ paraquat $(0,3 \mathrm{~kg} / \mathrm{ha}), 9)$ diquat $(0.3 \mathrm{~kg} / \mathrm{ha})$ e 10$)$ testemunha (colhida na época normal de colheita). Foram avaliadas a produtividade, o peso de 1000 sementes, a incidência de danos mecânicos no tegumento, a germinação, o vigor (envelhecimento natural) e a emergência no campo. Concluiu-se que: 1) os produtos dessecantes utilizados, independentemente das doses empregadas, não interferiram na produtividade nem na germinação das sementes de soja; 2) as sementes oriundas das plantas que foram dessecadas pelos produtos que atuam por contato apresentaram maior vigor que as oriundas das plantas dessecadas com o produto sistêmico; 3) apesar dos produtos não terem proporcionado a obtenção de sementes de melhor qualidade, a utilização dos mesmos poderá evitar, quando as condições climáticas forem adversas, a deterioração em campo, uma vez que antecipou a colheita em seis dias.
\end{abstract}

Palavras-chave: Glycine max, glyphosate, paraquat, diquat.

\section{ABSTRACT \\ Effects of desiccants aplication on the production and quality of soybean seeds in Uberlândia-MG, Brazil}

Field and laboratory experiments were used to test the effect of selected desiccants on the productivity and quality of soybean seeds, Savana cultivar. In the field ten treatments were arranged in a randomized complete block design, with four replications. Treatments were: (1) control (harvested during physiological maturation), (2) glyphosate-360 (0.36 kg/ha), (3) glyphosate-360 (0.72 kg/ha), (4) glyphosate-720 (0.36 kg/ha), (5) glyphosate-720 (0.72 kg/ha), (6) glyphosate-480 (0.36 kg/ha), (7) glyphosate-480 $(0.72 \mathrm{~kg} / \mathrm{ha})$, (8) paraquat $(0.30 \mathrm{~kg} / \mathrm{ha}),(9)$ diquat $(0.30 \mathrm{~kg} / \mathrm{ha})$, and (10) control (harvested during normal harvesting time). The treatments were evaluated for productivity, weight of 1000 seeds, mechanical damage of the integument, germination, vigor (natural aging) and field germination. Desiccants, at all rates, did not affect productivity and germination of soybean seeds. Seeds originated from plants desiccated by contact herbicides showed best vigor than those desiccated by sistemic herbicides. The desiccants did not improve seed quality, but their utilization could avoid, in adverse climate conditions, seed deterioration in the field, since the harvesting is antecipated in six days. 


\section{INTRODUÇÃO}

A expansão da cultura da soja deve-se, entre outras causas, ao uso de uma tecnologia mais apropriada ao processo produtivo, que vai desde a utilização de cultivares mais adequados até a fase final de uma segura e eficiente comercialização. No entanto, mesmo que se utilize uma tecnologia adequada, muitas vezes as condições climáticas não favorecem a um desenvolvimento satisfatório; surgem infestações de plantas daninhas na lavoura, alterações no ciclo vegetativo e imprevistos na fase de pós-maturação e colheita, entre outros fatores prejudiciais à qualidade da semente Gigax \& Burnside, 1976; Sanderson, 1976; Cathey, 1980; Carvalho \& Nakagawa, 1988).

Quando a semente atinge o máximo do peso da matéria seca, ela alcança, segundo Delouche (1975), o ponto de maturação fisiológica, ou seja, a semente se encontra no máximo de sua potencialidade e a deterioração é mínima (Carvalho \& Nakagawa, 1988). Entretanto após terem atingido a maturação, se não forem colhidas rapidamente, especialmente sob condições climáticas adversas, as sementes de soja entram em processo de deterioração, a qual pode abaixar a capacidade de germinação para níveis inaceitáveis.

De acordo com os dados de pesquisa, as sementes atingem a maturidade fisiológica antes que seu teor de umidade e o das plantas estejam baixos o suficiente para permitir que a colheita se processe mecanicamente. Andrews (1966) e Jacinto \& Carvalho (1974), por exemplo, trabalhando com diferentes cultivares de soja, em diferentes regiões (o primeiro nos EUA e os últimos no Brasil), verificaram que a maturidade fisiológica das sementes (equacionada com o máximo de matéria seca) é atingida quando o teor de umidade das sementes é de aproximadamente $50 \%$. Uma colheita feita depois deste ponto poderia, a rigor, ser considerada como feita em atraso, e isto, como já foi também documentado pela pesquisa, pode provocar severa redução na qualidade da semente.

Seria portanto, de grande vantagem que se pudesse realizar a colheita no ponto de maturação fisiológica, ou o mais próximo possível deste. Acontece, porém, que neste ponto, não somente o teor de umidade das sementes é muito elevado, como a própria planta ainda se encontra com grande número de folhas verdes, o que tornaria impossível o funcionamento da colhedora. Assim, uma alternativa para o produtor de sementes seria a utilização de um produto químico eficaz, de ação dessecante, no ponto de maturação fisiológica, permitindo a antecipação da colheita das sementes (Silva \& Silva, 1983; Carvalho \& Nakagawa, 1988). Os trabalhos realizados com soja, geralmente tem demonstrado a possibilidade de antecipação da colheita, dependendo da época e da efetividade da aplicação dos dessecantes (Springett, 1965; Obrador, 1966; Bastidas et al.,1971; Sanderson, 1976; Carvalho et al., 1978; Whigham \& Stoller, 1979; Batista Filho \& Barros, 1980).
Este trabalho foi conduzido com o objetivo de avaliar os efeitos da aplicação e de doses de diferentes produtos dessecantes na produção e qualidade das sementes da soja.

\section{MATERIAL E MÉTODOS}

Foram conduzidos dois ensaios, o primeiro em campo e o segundo em laboratório, para avaliar a produtividade e a qualidade das sementes respectivamente.

O ensaio de campo foi conduzido na Fazenda Alvorada, em uma área de produção comercial de sementes de soja da cultivar Savana, na safra 1991/92, em Uberlândia, MG. O delineamento experimental utilizado foi o de blocos casualizados, com quatro repetições, e os tratamentos constituídos de diferentes produtos e doses, além dos tratamentos utilizados como testemunha, discriminados na Tabela 1. As parcelas constituíram-se de oito fileiras de seis metros cada, espaçadas de 0,45 m, com área útil de 5,0 m x 0,9 m (duas fileiras centrais reduzidas em $0,5 \mathrm{~m}$ em cada extremidade).

O experimento foi instalado após a cultura estabelecida, sendo a população ajustada a 360 mil plantas por hectare. A dessecação foi realizada quando as plantas atingiram a maturação fisiológica detectada por indicadores visuais, conforme proposto por Crookston \& Hill (1978).

A partir da dessecação foi realizado o acompanhamento do declínio da umidade, sendo efetuada a colheita quando a umidade das sementes atingiu $13 \%$, exceto o tratamento que foi colhido no momento da dessecação, no qual as plantas secas foram colocadas ao sol, até atingirem a umidade de $13 \%$.

Após colhidas as sementes foram armazenadas em embalagens de pano, no ambiente do Laboratório de Análise de Sementes do Departamento de Agronomia da Universidade Federal de Uberlândia, até a colheita do último tratamento, quando foram avaliadas a produtividade, o peso de 1000 sementes, a incidência de danos mecânicos no tegumento, a germinação, a emergência em campo e o vigor.

A produtividade foi avaliada após o beneficiamento, pesando-se as sementes em balança com precisão de $1 \mathrm{~g}$. Os dados foram corrigidos para $10 \%$ de umidade e posteriormente transformados em $\mathrm{kg} / \mathrm{ha}$.

O peso de 1000 sementes foi realizado conforme especificado nas Regras para Análise de Sementes (Brasil, 1992). Os valores obtidos foram corrigidos para $10 \%$ de umidade. A incidência de dano mecânico no tegumento foi avaliada pelo método da imersão em hipoclorito de sódio (5\%), conforme Marcos Filho et al. (1987). O teste de germinação foi realizado de acordo com as prescrições das Regras para Análise de Sementes, (Brasil, 1992), com algumas modificações, conforme recomendações de Krzyzanowski et al. (1991). 
Tabela 1. Nome comum, nome comercial, doses e as testemunhas que compuseram os tratamentos avaliados. UberlândiaMG, 1995/96.

\begin{tabular}{|c|c|c|c|c|}
\hline \multicolumn{2}{|c|}{ Produto } & \multirow[b]{2}{*}{ Formulação } & \multicolumn{2}{|c|}{ Dose } \\
\hline Nome comum & Nome comercial & & $\begin{array}{c}\text { Ingrediente Ativo } \\
(\mathrm{kg} / \mathrm{ha})\end{array}$ & $\begin{array}{c}\text { Produto Comercial } \\
\text { (/ha) }\end{array}$ \\
\hline Glyphosate-360 & Roundup & $360 \mathrm{~g} / \mathrm{l}$ & 0,36 & 1,001 \\
\hline Glyphosate-360 & Roundup & $360 \mathrm{~g} / 1$ & 0,72 & 2,001 \\
\hline Glyphosate-780 & Direct & $720 \mathrm{~g} / \mathrm{kg}$ & 0,36 & 1,001 \\
\hline Glyphosate-720 & Direct & $720 \mathrm{~g} / \mathrm{kg}$ & 0,72 & 1,001 \\
\hline Glyphosate-540 & Rodeo + esp. $0.5 \%^{1}$ & $540 \mathrm{~g} / \mathrm{l}$ & 0,36 & 0,751 \\
\hline Glyphosate-540 & Rodeo + esp. $0.5 \%^{1}$ & $540 \mathrm{~g} / 1$ & 0,72 & 1,501 \\
\hline Paraquat & Gramoxone + esp. $0.5 \%{ }^{1}$ & $200 \mathrm{~g} / 1$ & 0,30 & 1,501 \\
\hline Diquat & Reglone + esp. $0.5 \%^{1}$ & $200 \mathrm{~g} / \mathrm{l}$ & 0,30 & 1,501 \\
\hline
\end{tabular}

$\begin{array}{lll}\text { Testemunhas: } & \text { 1. Colhidas na maturação fisiológica } \quad \text { 2. Colhidas na época normal }\end{array}$

' Utilizou-se como espalhante adesivo o Adafix (Ácido sulfônico dodecil benzeno 300 g/1 + carboximeticulose de sódio 300 g/l); solução aquosa; grupo químico: sal do ácido sulfônico dodecilbenzeno.

O estudo da emergência em campo foi realizado na área experimental da Universidade Federal de Uberlândia, campus Umuarama. Neste teste adotou-se o mesmo delineamento do experimento de campo que deu origem às sementes em estudo. As parcelas constituíram-se de quatro sulcos de 1,75 m cada, espaçados de 0,5 m, semeando-se 30 sementes por metro de sulco aberto manualmente em solo arado e gradeado, à profundidade de $0,03 \mathrm{~m}$ e adubado utilizando-se $500 \mathrm{~kg} /$ ha do adubo formulado 2-20-20. Na determinação da percentagem de plântulas emersas, foi considerado o número total de plântulas aos 14 dias após a semeadura, transformando-se em percentagens.

O vigor foi avaliado após o envelhecimento natural das sementes, as quais foram armazenadas por 12 meses em condições de ambiente no laboratório. Após este período, foram submetidas ao teste de germinação.

\section{RESULTADOS E DISCUSSÃO}

Observa-se que, de maneira geral, as produtividades obtidas se encontram na média da região e que não houve interferência da dessecação na produção (Tabela 2). Entretanto, cuidados deverão ser tomados na definição do ponto de maturação, uma vez que, caso a dessecação química seja realizada antes que a planta atinja a maturação fisiológica, ocorre queda na produção (Whigham \& Stoller, 1979).

Para o peso de 1000 sementes, embora não tenha diferido significativamente a $5 \%$ pelo teste de Tuckey, verifica-se que o maior valor foi obtido quando as sementes foram colhidas na maturação fisiológica, enquanto que as sementes colhidas no ponto de colheita apresentaram o menor valor (Tabela 3). De um modo geral, a variação entre tratamen- tos foi pequena. Este fato confirma a tendência dos dados de produtividade e também demonstra que a maturação fisiológica foi bem definida, apesar de ter sido considerada quando $90 \%$ das vagens já tinham atingido este ponto, concordando com os resultados de Batista Filho \& Barros (1980).

Tabela 2. Valores médios da produtividade de semente de soja provenientes de plantas submetidas aos diversos tratamentos com dessecantes. Uberlâdia-MG, 1995/96.

\begin{tabular}{lcc}
\hline Tratamento & $\begin{array}{c}\text { Dose } \\
\text { (kg/ha) }\end{array}$ & $\begin{array}{c}\text { Produção } \\
\text { (kg/ha) }\end{array}$ \\
\hline Maturação Fisiológica & - & $2070,2 \mathrm{a}^{1}$ \\
Glyphosate-360 & 0,36 & $2042,9 \mathrm{a}$ \\
Glyphosate-360 & 0,72 & $2046,1 \mathrm{a}$ \\
Glyphosate-780 & 0,36 & $2060,4 \mathrm{a}$ \\
Glyphosate-720 & 0,72 & $2049,1 \mathrm{a}$ \\
Glyphosate-540 & 0,36 & $2008,6 \mathrm{a}$ \\
Glyphosate-540 & 0,72 & $2041,7 \mathrm{a}$ \\
Paraquat & 0,30 & $1985,2 \mathrm{a}$ \\
Diquat & 0,30 & $1998,7 \mathrm{a}$ \\
Época Normal de colheita & - & $1936,3 \mathrm{a}$ \\
\hline
\end{tabular}

${ }^{1} \mathrm{Na}$ coluna, as médias seguidas por uma mesma letra não diferem significativamente a $5 \%$ de probabilidade, pelo teste de Tukey.

Com relação ao dano mecânico no tegumento, observa-se que os tratamentos que apresentaram maior incidência de danos foram aqueles cujos produtos eram à base de paraquat e diquat, os quais são herbicidas de contato, enquanto que nos tratamentos que receberam os produtos à base de glyphosate, que é sistêmico, houve uma menor incidência 
Tabela 3. Valores médios do peso 1000 sementes, dano no tegumento e grau de umidade na maturação fisiológica e no ponto de colheita das sementes de soja, oriundas de plantas submetidas aos diversos tratamentos com dessecantes. Uberlândia-MG, 1995/96.

\begin{tabular}{|c|c|c|c|c|c|c|}
\hline \multirow{2}{*}{ Tratamento } & \multirow{2}{*}{$\begin{array}{c}\text { Dose } \\
\text { (kg/ha) }\end{array}$} & \multirow{2}{*}{$\begin{array}{l}\text { Peso de } 1000 \\
\text { sementes }(\mathrm{g})\end{array}$} & \multirow{2}{*}{$\begin{array}{c}\text { Dano no } \\
\text { tegumento } \\
(\%)\end{array}$} & \multicolumn{2}{|c|}{ Grau de umidade em base úmida (\%) } & \multirow{2}{*}{$\begin{array}{l}\text { Data de } \\
\text { colheita }\end{array}$} \\
\hline & & & & $\begin{array}{l}\text { Maturação } \\
\text { Fisiológica }\end{array}$ & Ponto de Colheita & \\
\hline Maturação Fisiológica & - & $145,8 \mathrm{a}^{1}$ & $34,0 \mathrm{~b}^{1}$ & $38,3 \mathrm{a}^{1}$ & - & $09 / 03$ \\
\hline Glyphosate-360 & 0,36 & $141,7 \mathrm{a}$ & $34,1 \mathrm{~b}$ & $37,1 \mathrm{a}$ & $13,5 \mathrm{a}^{1}$ & $24 / 03$ \\
\hline Glyphosate-360 & 0,72 & $138,4 \mathrm{a}$ & $34,9 \mathrm{~b}$ & 39,6 a & $14,2 \mathrm{a}$ & $24 / 03$ \\
\hline Glyphosate-780 & 0,36 & $141,1 \mathrm{a}$ & $35,8 \mathrm{~b}$ & $36,5 \mathrm{a}$ & $14,0 \mathrm{a}$ & $23 / 03$ \\
\hline Glyphosate-720 & 0,72 & $142,2 \mathrm{a}$ & $36,6 \mathrm{~b}$ & $36,0 \mathrm{a}$ & $14,3 \mathrm{a}$ & $23 / 03$ \\
\hline Glyphosate-540 & 0,36 & $139,5 \mathrm{a}$ & $34,1 \mathrm{~b}$ & $36,9 \mathrm{a}$ & $13,9 \mathrm{a}$ & $24 / 03$ \\
\hline Glyphosate-540 & 0,72 & $142,2 \mathrm{a}$ & $35,7 \mathrm{~b}$ & $37,1 \mathrm{a}$ & $13,7 \mathrm{a}$ & $24 / 03$ \\
\hline Paraquat & 0,30 & $138,6 \mathrm{a}$ & $40,3 \mathrm{a}$ & $37,2 \mathrm{a}$ & $13,7 \mathrm{a}$ & $23 / 03$ \\
\hline Diquat & 0,30 & $138,3 \mathrm{a}$ & 39,4 a & $36,6 \mathrm{a}$ & $13,6 \mathrm{a}$ & $23 / 03$ \\
\hline Época Normal de Colheita & - & $134,3 \mathrm{a}$ & $36,3 \mathrm{~b}$ & $39,8 \mathrm{a}$ & $14,9 \mathrm{a}$ & $30 / 03$ \\
\hline
\end{tabular}

${ }^{1} \mathrm{Na}$ coluna, as médias seguidas por uma mesma letra não diferem significativamente a $5 \%$ de probabilidade, pelo teste de Tukey.

de dano no tegumento, provavelmente pelo declínio de umidade ter ocorrido mais lentamente, em função da secagem menos drástica das plantas (Tabela 3).

Fraga (1988), verificou que o paraquat, quando aplicado isoladamente, promoveu um dessecamento mais rápido do que quando aplicado em associação com o diuron. Já Austin (1972) e Andreoli \& Ebeltoft (1979), notaram que doses e épocas de aplicação dos dessecantes tiveram efeitos na redução da umidade da semente de ambas as cultivares que trabalharam, entretanto, diferenças nas taxas de secagem não foram observadas entre os herbicidas glyphosate e paraquat.

Na maturação fisiológica, época em que foram aplicados os tratamentos, a umidade era aproximadamente a mesma para todas as parcelas que receberam os tratamentos, variando de 35 a $39 \%$ (Tabela 3 ). Este resultado já era esperado, por tratar-se de um campo de produção comercial de soja muito homogêneo e as plantas ainda não tinham sido submetidas a nenhum tratamento (Fonseca, 1984).

Com relação ao grau de umidade no ponto de colheita, verifica-se que todos os tratamentos que receberam os dessecantes estavam em condições para serem colhidos praticamente no mesmo dia, sendo que a testemunha ainda não apresentava tal condição, permanecendo no campo por mais de seis dias, até apresentar um grau de umidade ideal para ser colhida.

Estes resultados mostram que a dessecação antecipou em seis dias a colheita das sementes, estando de acordo com Sampson \& Menendez (1979) e Andreoli \& Ebeltoft (1979), os quais afirmaram que a dessecação acelera a desidratação das sementes, fazendo com que estas fiquem menos expostas às variações do ambiente, permitindo desta forma a obten- ção de sementes de melhor qualidade. Resultado semelhante foi obtido por Batista Filho \& Barros (1980) e por Durigan $\&$ Carvalho (1980), que utilizando dessecantes conseguiram antecipar a colheita da soja.

Com relação à germinação observou-se que as sementes colhidas na maturação fisiológica sobressaíram em relação às demais (Tabela 4). Para todos os tratamentos os valores obtidos estão acima do índice mínimo de germinação exigido pela Comissão Estadual de Sementes e Mudas para Minas Gerais, que é de 75\% (Minas Gerais, 1992). Embora não tenha ocorrido diferença significativa verifica-se, pelos resultados da germinação, que a dessecação possibilitou a obtenção de sementes com uma maior taxa quando comparada com as colhidas em época normal. Costa et al. (1983) também verificaram diferenças muito pequenas entre os tratamentos dessecados e a testemunha, sendo que, em alguns casos, não houve diferença.

$\mathrm{O}$ tratamento que teve a maior emergência em campo foi colhido na maturação fisiológica (Tabela 4). Os demais não diferiram entre si, demonstrando que a dessecação não afetou o potencial da emergência no campo.

Estes resultados mostram que as sementes, quando colhidas no ponto de maturação fisiológica, apresentam maior vigor, ou seja, quando são submetidas a alguma condição adversa em campo, sobressaem em relação às demais. Segundo Carvalho \& Nakagawa (1988), na maturação fisiológica as sementes se encontram no máximo de sua potencialidade, sendo mínima a sua deterioração.

Com relação ao vigor, avaliado após o envelhecimento natural das sementes por 12 meses, verifica-se que os tratamentos que receberam os dessecantes que atuam por con- 
tato (paraquat e diquat), apresentaram maior germinação, não diferindo das sementes colhidas na maturação fisiológica, sugerindo que estes produtos afetaram em menor grau a qualidade das sementes ao longo do armazenamento. Tais resultados estão de acordo com Wigham \& Stoller (1979), que não recomendam glyphosate como dessecante, pois este diminuiu o vigor das sementes, sendo que produtos à base de paraquat não alteraram o vigor e a germinação das mesmas.

Tabela 4. Valores médios da percentagem de germinação de sementes e de plântulas emersas em campo, provenientes de plantas de soja submetidas aos diversos tratamentos com dessecantes. Uberlândia-MG, 1995/96.

\begin{tabular}{lccc}
\hline Tratamento & Dose & $\begin{array}{c}\text { Germi- } \\
\text { nação } \\
\mathbf{( \% )}\end{array}$ & $\begin{array}{c}\text { Emer- } \\
\text { gência em } \\
\text { campo } \\
\mathbf{( \% )}\end{array}$ \\
\hline Maturação Fisiológica & - & $85,5 \mathrm{a}^{1}$ & $79,5 \mathrm{a}^{1}$ \\
Glyphosate-360 & 0,36 & $78,3 \mathrm{~b}$ & $67,5 \mathrm{~b}$ \\
Glyphosate-360 & 0,72 & $78,7 \mathrm{~b}$ & $60,0 \mathrm{~b}$ \\
Glyphosate-780 & 0,36 & $78,6 \mathrm{~b}$ & $68,5 \mathrm{~b}$ \\
Glyphosate-720 & 0,72 & $78,3 \mathrm{~b}$ & $58,7 \mathrm{~b}$ \\
Glyphosate-540 & 0,36 & $77,6 \mathrm{~b}$ & $59,3 \mathrm{~b}$ \\
Glyphosate-540 & 0,72 & $78,5 \mathrm{~b}$ & $59,0 \mathrm{~b}$ \\
Paraquat & 0,30 & $79,9 \mathrm{~b}$ & $63,5 \mathrm{~b}$ \\
Diquat & 0,30 & $79,0 \mathrm{~b}$ & $64,0 \mathrm{~b}$ \\
Época Normal de Colheita & - & $74,7 \mathrm{~b}$ & $61,0 \mathrm{~b}$ \\
\hline
\end{tabular}

' Na coluna, as médias seguidas por uma mesma letra não diferem significativamente a $5 \%$ de probabilidade, pelo teste de Tukey.

Tabela 5. Valores médios da percentagem de germinação, obtidos após o envelhecimento natural das sementes de soja por 12 meses, provenientes de plantas submetidas aos diversos tratamentos com dessecantes. Uberlândia-MG, 1995/96.

\begin{tabular}{lcc}
\hline \multicolumn{1}{c}{ Tratamento } & Dose & Germinação (\%) \\
\hline Maturação Fisiológica & - & $69,5 \mathrm{a}^{1}$ \\
Glyphosate-360 & 0,36 & $56,5 \mathrm{~b}$ \\
Glyphosate-360 & 0,72 & $56,0 \mathrm{~b}$ \\
Glyphosate-780 & 0,36 & $55,5 \mathrm{~b}$ \\
Glyphosate-720 & 0,72 & $50,0 \mathrm{~b}$ \\
Glyphosate-540 & 0,36 & $50,5 \mathrm{~b}$ \\
Glyphosate-540 & 0,72 & $50,0 \mathrm{~b}$ \\
Paraquat & 0,30 & $63,5 \mathrm{a}$ \\
Diquat & 0,30 & $64,0 \mathrm{a}$ \\
Época Normal de Colheita & - & $51,0 \mathrm{~b}$ \\
\hline
\end{tabular}

${ }^{1} \mathrm{Na}$ coluna, médias seguidas por uma mesma letra não diferem significativamente a $5 \%$ de probabilidade, pelo teste de Tukey.

\section{CONCLUSÕES}

Os produtos dessecantes glyphosate, paraquat e diquat, independentemente das doses empregadas, não interferiram na produtividade nem na germinação das sementes de soja.

As sementes oriundas das plantas que foram dessecadas pelos produtos que atuam por contato (paraquat e diquat), apresentaram maior vigor que as oriundas das plantas dessecadas com o produto sistêmico (glyphosate). Apesar dos produtos não terem proporcionado a obtenção de sementes de melhor qualidade, a utilização dos mesmos poderá evitar, quando as condições climáticas forem adversas, a deterioração em campo, uma vez que antecipou a colheita em seis dias.

\section{LITERATURA CITADA}

ANDREOLI, C.G; EBELTOFT, D.C. Dessecantes no rendimento e na qualidade das sementes de soja. Brasília, Pesq. Agropc. Bras., v. 14, n. 2, p. 135-139. 1979.

ANDREWS, C. H. Some aspects of pod and seed development in Lee soybeans. Mississipi, Mississipi State University, 1966. 75 p. (Tese PhD).

AUSTIN, R. B. Effect of environment before harvesting on viability. In: ROBERTS, E. H. Viability of seeds. Syracuse: Syracuse University Press, 1972. p. 114-119.

BASTIDAS, G. H.; FRANCO, H.; CRUZ, R. Desfoliantes en soya. Acta Agronomica, v. 21, p. 51-58. 1971.

BATISTA FILHO, O. C.; BARROS, A C. Efeitos de dessecantes na maturação e qualidade de sementes de soja (Glycine max (L.) Merrill). Tecnologia Sementes, v. 3 , n. 2, p. 19-26. 1980

BRASIL, MINISTÉRIO DA AGRICULTURA. Ministério da Agricultura e Reforma Agrária. Regras para análise de sementes. Brasília: SNDA/DNDV/CLAV, 1992. $365 \mathrm{p}$.

CARVAlHO, N. M.; NAKAGAWA, J. Sementes, ciência, tecnologia e produção. Campinas: Fundação Cargil, 1988. $424 \mathrm{p}$.

CARVALHO, N.M.; DURIGAN, J. C.; BARRETO, M.; DURIGAN, J. F. Aplicação pré-colheita de dessecante de soja (Glycine max (L) Merrill) do cultivar Viçoja. 1. Efeitos sobre a produção de grãos. Científica, v. 6, n.2, p. 75-79. 1978.

CATHEY, G. H. Harvest-aid chemicals and practices for cotton. Outlook on Agriculture, v.10, n. 4, p. 191-197. 1980. 
COSTA, N. P.; FRANÇA NETO, J. B.; PEREIRA, L. G.; HENNING, A. A; TURKIEWICZ, L.; DIAS, M. C. L. Antecipação de colheita de sementes de soja através do uso de dessecantes. Rev. Bras. Sem, v. 5, n. 3, p. 181198. 1983

CROOKSTON, R. K. \& Hill, D. S. A visual indicator of the physiological maturity of soybean seed. Crop. Sci., v. 18, n. 5 , p. $867-870.1978$.

DELOUCHE, J. C. Recentes conquistas da pesquisas tecnológica de sementes. In: DELOUCHE, J. C. Pesquisa em sementes no Brasil. Brasília: AGIPLAN, 1975. p. 27-36.

DURIGAN, J. F. \& CARVALHO, N. M. Aplicação em précolheita de dessecantes em duas cultivares de soja (Glycine max (L.) Merrill). 1. Efeitos imediatos sobre a germinação e produção de sementes. Planta Daninha, v. 3, n. 2, p. 108-15,1980.

FONSECA, N. Influência da aplicação de paraquat sobre a produção e a qualidade da semente de soja (Glycine $\max (\mathrm{L}$.$) Merrill). Viçosa, Universidade Federal de Vi-$ çosa. 1984.48 p. (Tese MS).

FRAGA. A. C. Estudo sobre a utilização de dessecantes na produção de sementes de soja (Glycine max (L.) Merrill). Viçosa, Universidade Federal de Viçosa. 1988. 91 p. (Tese DS).

GIGAX, D. R.; BURNSIDE, O. C. Chemical desiccation of grain sorghum. Agron. J., v. 68, n. 4, p. 645-9. 1976.

KRZYZANOWSKI, F. C.; FRANCA NETO, J. B.; HENNING, A. A. Relatos dos testes de vigor disponíveis para as grandes culturas. Inf. Abrates Brasília, v. 1, n. 2, p. 15-50, 1991.
JACINTO, J. B. C.; CARVALHO, N. M. Maturação de sementes de soja. Científica, v. 1, n. 1, p. 81-88. 1974.

MARCOS FILHO, J.; CICERO, S. M.; SILVA, W. R. Avaliação da qualidade das sementes. Piracicaba, FEALQ, 1987. $230 \mathrm{p}$.

MINAS GERAIS. Secretaria de Agricultura. Comissão Estadual de Sementes e Mudas. Normas, padrões e procedimentos para produção de sementes básicas, certificadas e fiscalizadas. Belo Horizonte, 1992. 110p.

OBRADOR, J. R. Dessecantes en producción de semilla de plantas forrageiras. Agricultura Técnica, v. 26, n. 4, p. 164-168. 1966.

SANDERSON, J. F. Pre-harvest desiccation of oil seed crops. Outlook on Agriculture, v. 9, n. 1, p. 21-25. 1976.

SAMPSON, A. A G.; MENENDEZ, A. C. Dessecação de soja. In: Seminário Nacional de Pesquisa de soja, 1. Londrina, 1978. Anais..., Londrina, EMBRAPA/ CNPSo, vol. 2. p.169-178. 1979.

SILVA, R. F.; SILVA, J. F. O uso de dessecantes na produção de sementes de soja. In: Universidade Federal de Viçosa. Dia de campo na CEPET. Capinópolis: Universidade Federal de Viçosa, 1983. p. 70.

SPRINGETT, R. H. The bipyridylium herbicides: their properties and use. Outlook on Agriculture, v. 4, n. 5, p. 226-233. 1965.

WHIGHAM, D. K.; STOLLER, E. W. Soybean desiccation by paraquat, glyphosate and ametryn to accelerate harvest. Agron. J., v. 71, p. 630-633. 1979. 